\title{
THE TRACE FORMULA FOR VECTOR BUNDLES
}

\section{GUILLEMIN AND A. URIBE}

Let $X$ be a compact Riemannian manifold and let $0=\lambda_{1}<\lambda_{2} \leq \lambda_{3} \leq \cdots$ be the spectrum of the Laplace operator. By a theorem of Hermann Weyl the spectral counting function

$$
N(\lambda)=\#\left\{\lambda_{i}^{2}<\lambda\right\}
$$

satisfies a growth estimate of the form $O\left(\lambda^{N}\right)$, so its Fourier-Stieltjes transform

$$
\int e^{i \lambda t} d N(\lambda)=\sum_{k} e^{ \pm i \sqrt{\lambda_{k} t}}
$$

is a tempered distributional function of $t$. The classical trace formula says that the singular support of (II) is contained in the length spectrum of $X$. Moreover, under suitable hypotheses on geodesic flow, the trace formula gives considerable information about the singularities in (II). (See [DG and C].)

There is a fairly straightforward (and not terribly interesting) generalization of the trace formula to vector bundles. (See, for instance, the introduction to [DG].) We will be concerned in this article with a much more subtle generalization inspired by recent articles of Hogreve, Potthoff, and Schrader [HPS], and Schrader and Taylor [ST] in Communications in Mathematical Physics.

Let $G$ be a compact Lie group and $\pi: P \rightarrow X$ a principle $G$-bundle with connection. Given a finite-dimensional unitary representation, $\rho$, of $G$ we will denote by $E \rho$ the vector bundle over $X$ associated with $\rho$ and by $D_{\rho}$ the associated connection.

Now consider a ladder $\left\{\rho_{e}, e=1,2, \ldots\right\}$ of irreducible representations of $G$. (This means that the maximal weight of $\rho_{e}$ is $e$ times the maximal weight of $\rho_{1}$.) For given $e$ let

$$
\lambda_{k, e}, \quad k=1,2,3, \ldots
$$

be the spectrum of the Laplace operator on $C^{\infty}\left(E \rho_{e}\right)$ :

$$
\Delta_{e}=D^{*} \rho_{e} D \rho_{e}+e^{2} \text {. }
$$

The Hogreve-Potthoff-Schrader and Schrader-Taylor papers are concerned with asymptotic properties of the quantities $e$ and $E=\lambda_{k, e}$ when $e$ and $E$ tend to infinity in such a way that the ratio $r=e: \sqrt{E}$ is (approximately) constant. One way to measure such asymptotic behavior is as follows: Fix a Schwartz function of one variable, $\varphi(s)$, with $\varphi(s) \geq 0$ and $\int \varphi(s) d s=1$, and form the sum

$$
N_{\varphi, r}(\lambda)=\sum_{e} \sum_{\lambda_{k, e}<\lambda^{2}} \varphi\left(r \sqrt{\lambda_{k, e}}-e\right) .
$$

Received by the editors May 7, 1986.

1980 Mathematics Subject Classification (1985 Revision). Primary 58G25. 
This sum is similar to the sum (I) except that it counts eigenvalues in such a way that eigenvalues for which the ratio $e: \sqrt{E}$ is close to $r$ are weighted much more heavily than eigenvalues for which the ratio is far away from $r$. Our main result is a trace formula for the Fourier-Stieltjes transform of $N_{\varphi, r}$. To formulate it we need to recall some facts about the coupling of a classical dynamical system to a Yang-Mills field. Let $O_{e}$ be the co-adjoint orbit in $g^{*}$ associated with the representation, $\rho_{e}$, and let $M_{e}$ be the symplectic manifold obtained by reducing $T^{*} P$ with respect to $O_{e}$. By a theorem of Sternberg [S] and Weinstein $[\mathbf{W}]$ the choice of a connection on $P$ gives rise to a symplectic fiber mapping

$$
\pi_{e}: M_{e} \rightarrow T^{*} X
$$

If $H$ is the standard Kinetic energy Hamiltonian on $T^{*} X$ describing the motion of a classical particle on $X$ when no background field is present, $\pi_{e}{ }^{*} H$ describes the motion of a classical particle (of "charge" $e$ ) when a background field is present. (See $[\mathbf{S}]$.)

Now fix $e$ and $E$ so that $e: \sqrt{E}=r$ and consider the restriction to the energy surface $\pi_{e}{ }^{*} H=E$ of the Hamiltonian system on $M_{e}$ associated with $\pi_{e}{ }^{*} H$. We will assume that the periodic trajectories of this system are nondegenerate and denote the set of these trajectories by $\Gamma$.

THEOREM. If the function $\hat{\varphi}$ has compact support, the Fourier-Stieltjes transform of $N_{\varphi, r}(\lambda)$ can be written as a locally finite sum

$$
e_{0}(t)+\sum_{\gamma \in \Gamma} e_{\gamma}(t)
$$

where $e_{0}$ and the $e_{\gamma}$ 's are distributions of compact support. Moreover, $e_{0}$ has no singularities except for a classical conormal singularity at the origin, and $e_{\gamma}$ has no singularities except for a classical conormal singularity at $t=T_{\gamma}+r \theta_{\gamma}$. Here $T_{\gamma}$ is the period of $\gamma$ and $\theta_{\gamma}$ an appropriate determination of

$$
\frac{\log (\text { holonomy of } \gamma)}{2 \pi i}-r T_{\gamma}
$$

Moreover, $e_{\gamma}(t)$ is equal to

$$
c_{\gamma} \delta\left(t-T_{\gamma}-r \theta \gamma\right)+\tilde{e}_{\gamma}(t)
$$

where $\tilde{e}_{\gamma}(t)$ is in $\mathcal{L}^{1}$ and the constant $c_{\gamma}$ is related to the primitive period $T_{\gamma}^{*}$ of $\gamma$ and the linearized Poincaré map, $P_{\gamma}$, by the formula

$$
c_{\gamma}=\hat{\varphi}\left(\theta_{\gamma}\right) \frac{T_{\gamma}^{*}}{2 \pi} \frac{\exp \left(i \pi \sigma_{\gamma} / 4\right)}{\left|I-P_{\gamma}\right|^{1 / 2}}
$$

$\sigma_{\gamma}$ being a suitable Maslov index.

REMARKs. (1) There is an analogue of (VI) for $e_{0}(t)$ as well, which we won't both to describe here. From it one gets a Weyl formula for the asymptotic behavior of $N_{\varphi, r}(\lambda)$.

(2) Just as in the classical case, an analogue of the expansion (V) is true when the periodic trajectories on the $\pi_{e}{ }^{*} H=E$ energy surface form "clean" submanifolds. (See $[\mathbf{D G}, \S 6]$.) 


\section{BIBLIOGRAPHY}

[C] J. Chazarain, Formule de Poisson pour les variétés riemanniennes, Invent. Math. 24 (1974), 65-82.

[DG] J. J. Duistermaat and V. Guillemin, The spectrum of positive elliptic operators and periodic geodesics, Invent. Math. 29 (1975), 184-269.

[HPS] H. Hogreve, J. Potthoff and R. Schrader, Classical limits for quantum particles in external Yang-Mills potentials, Comm. Math. Phys. 91 (1983), 573-598.

[ST] R. Schrader and M. Taylor, Small $h$ asymptotics for quantum partition functions associated to particles in external Yang-Mills potentials, Comm. Math. Phys. 92 (1984), 555-594.

[S] S. Sternberg, On minimal coupling and the symplectic mechanics of a classical particle in the presence of a Yang-Mills field, Proc. Nat. Acad. Sci. U.S.A. 74 (1977), 5253-5254.

[W] A. Weinstein, A universal phase space for particles in Yang-Mills fields, Lett. Math. Phys. 2 (1978), 417-20.

Department of Mathematics, Massachusetts institute of TeChNOLOGY, CAMBRIDGE, MASSACHUSETTS 02139

Department of Mathematics, Princeton University, Princeton, New JERSEY 08540 